determine the seasonal rhythms, which in turn characterize the plant species making up the major structural elements of the major forest habitats. Against this background the reader is led through the manner in which arboreal and terrestrial frugivores utilize the forest's primary production, and the role of insects and arthropods which feed in the canopy and on ground litter. The final section will be of special interest to those who study similar conservation areas that are defined by distinct physical boundaries limiting the dispersal of elements of the flora and fauna. One of the editors, Egbert Leigh, provides a theoretical background to the two important studies of temporal variations in populations of birds and terrestrial mammal communities, with the latter revealing 29,000 of the larger components on 1500 ha, a high density of over 19 animals per hectare.

This volume will stand alongside $\mathrm{H}$. T. Odum and R. F. Pigeon's A Tropical Rain Forest (US Atomic Energy Commission, 1970) and A. R. E. Sinclair and M. NortonGriffiths's Serengeti - the Dynamics of an Ecosystem (Chicago University Press, 1979) as vital reading for biologists who wish to view tropical ecosystems as a whole. It may also encourage those funding tropical ecological research to initiate similar broad-based studies in other parts of the world, for the fashionable demand for environmental impact statements and management plans has little meaning without the collection of such basic data.

Malcolm Coe is a Lecturer in Animal Ecology in the Department of Zoology, University of Oxford.

\section{Anomalous science}

\section{V.E. Viola}

\section{The Origin of the Chemical Elements and} the Oklo Phenomenon.

By P.K. Kuroda.

Springer-Verlag: 1983. Pp.164. DM 92 $\$ 39.70$.

FASCINATING insights into the evolution of nature's elements and the subsequent history of our Solar System can be obtained from the study of elemental abundances and isotopic anomalies in terrestrial, meteoritic and lunar materials. In this book, Paul Kuroda examines some of the facets of this subject with which he has been involved in his distinguished research career.

The book has a distinct historical flavour, containing an extensive list of early references, but it also highlights several significant areas of current research in geo- and cosmochemistry. The title is somewhat misleading, however, as the emphasis is not so much on the origin of the elements as it is on the search for rare nuclear species in nature and the perturbations of the major nucleosynthetic processes that have produced observed anomalies in normal abundances.

The author introduces his subject by tracing the efforts to compile a uniform table of Solar System abundances. The account falls somewhat short, however, in that it does not extend to the inclusion of currently-used tables, nor does it examine the complexities inherent in generating such compilations. However, in Chapter Three attempts to detect ${ }_{43} \mathrm{Tc}$ and ${ }_{61} \mathrm{Pm}$, absent in nature due to their radioactivity, are discussed and this emphasizes the critical role of analytical sensitivity in establishing the existence of rare or anomalous isotopic species in natural materials.

The most significant contributions are found in Chapters Four and Six. The former deals with the Oklo phenomenon the extinct natural nuclear reactor discovered in the Republic of Gabon, in 1972. Beginning with the factors which led the author to predict the existence of such a reactor in 1956, both the theory of natural reactors and the experimental efforts to detect them are extensively discussed. Chapter Six focuses on the extinct radionuclides ${ }^{244} \mathrm{Pu}\left(t_{1 / 2}=8.1 \times 10^{7} \mathrm{y}\right)$ and ${ }^{129} \mathrm{I}\left(t_{1 / 2}=1.6 \times 10^{7} \mathrm{y}\right)$. In addition to describing attempts to detect primordial remnants of these radioactive species, the author stresses their important relationship to the interpretation of xenon isotope ratios in natural materials.

Theories of nucleosynthesis are dealt with in Chapter Five, the coverage of this topic being largely historical in nature, while in the final chapter the author proposes a unified theory to account for the unusual isotopic abundances found in certain meteorites (R.N. Clayton, et al., Science 182, 485; 1973). He begins by examining the origin of the rare elements $\mathrm{Li}, \mathrm{Be}$ and $\mathrm{B}$ and the meteoritic isotope anomaly data. (A major $\mathrm{Li}$ Be $\mathrm{B}$ reference is omitted from these discussions, S.M. Austin, Prog. Part. Nucl. Phys. 40, 14; 1980 ). Then it is postulated that the nuclear processes responsible for $\mathrm{Li}, \mathrm{Be}$ and $\mathrm{B}$ synthesis were instrumental in the creation of the isotopic anomalies observed in meteorites. However, in relating these two phenomena, the author assumes that all ${ }^{7} \mathrm{Li}$ in the Solar System is produced by cosmic ray interactions. Because only about $10 \%$ of Solar System ${ }^{7} \mathrm{Li}$ is presently believed to be of cosmic ray origin, this analysis is not compelling.

However, the chapter concludes with a summary of isotope anomalies in several elements, which serves as a useful indication of the complexity and future promise for intriguing results in this field.

V.E. Viola is Professor of Chemistry at Indian University, Bloomington.
Wild orchids

\section{M.A. Clements}

Wild Orchids of Britain and Europe.

By P. and J. Davies, and A. Huxley.

Chatto \& Windus/The Hogarth Press:

1983. Pp.304. £9.95.

AT first sight this book appears to be all that English-speaking orchid enthusiasts have been waiting for - an authoritative up-to-date account of European orchids. Indeed, it is claimed to be so by the authors. The photographs are excellent but, unfortunately, the text does them scant justice as it contains scientific inaccuracies, half truths and generalizations many of which are untrue. There are, in fact, errors on nearly every page of the book, some minor but others of a more serious nature. For example, in the introductory chapter there is a section on seed germination in which most of the information is out of date by some 30 years or more. The authors have also perpetuated errors that have existed in the literature for decades. For example available evidence from in vitro germination of orchid seeds shows that it takes but a few months and not years, as is suggested on pages 6 and 8 , for the first leaf of most orchids to emerge from the protocorm.

Several comments made in the text about particular name changes of orchids that the authors dislike illustrate their lack of understanding of the taxonomic method. For example, in the section on classification the reader might gain the impression that most botanists are incapable of correctly classifying and naming European orchids; in fact, the naming of plants, including orchids, is strictly controlled by the International Code of Botanical Nomenclature. Clearly the authors have little, if any, understanding of the rules governing the naming of plants. Thus, Ophrys holoserica is the only correct scientific name for the Late Spider Orchid. The authors prefer to use a name reduced to synonymy several years ago. Unfortunately, this is not an isolated example.

Scientific terms have been frequently misused in the text. In botany, it is more usual to speak of populations of plants rather than "races", a term more appropriate to zoology. Terms such as classes, tribes, genera, forms etc. are consistently misused, for example on page 8 where Ophrys and Orchis are referred to as tribes instead of genera.

By all means buy this book - its illustrations alone warrant it. But beware of accepting the often inaccurate opinions that accompany them.

M.A. Clements is on the Research Staff of the National Botanic Gardens, Australia, and currently an Associate Research Fellow at the Royal Botanic Gardens, Kew. 